\title{
Insole Program in the Manufacturing Setting: Impact on Lower Extremity Muscle Activity
}

\author{
Carley $\mathrm{P}^{1 *}$, Kessler J ${ }^{2}$ and Lachowski S1 \\ ${ }^{1}$ American International College, Springfield, MA, USA \\ ${ }^{2}$ Hasbro Games, East Longmeadow, MA, USA
}

*Corresponding author: Patrick Carley, Professor, Doctorate of Physical Therapy

Program, School of Health Sciences, American International College, Springfield, MA 01109, USA, Tel: 413-205-3294; Email: Patrick.Carley@aic.edu

\section{Research article \\ Volume 1 Issue 3}

Received Date: October 24, 2017

Published Date: November 10, 2017

DOI: $10.23880 /$ eoij-16000121

\section{Abstract}

Hasbro Games in East Longmeadow, Massachusetts sought to evaluate the impact of their year-long companyinstituted footwear insole program after removing floor mats. The employees subjectively reported reduced aches and pains while standing and working on concrete factory floors with the insoles; but because there was no objective data to substantiate their responses, the actual mechanism influencing the reported reductions in aches and pains remained unknown.

Hasbro and the American International College's physical therapy program collaboratively designed a study using electromyography (EMG) to investigate the potential difference in lower leg muscle activity with and without the insoles. Of the several employees who volunteered, 43 were randomly selected to participate in the EMG insole study. All participants reported standing or walking on concrete floors for 60 to 90 percent of their workday.

Results of the EMG study disclosed a consistent reduction in lower leg muscle activity for both of the muscle groups using the company-issued polyurethane insoles compared with no insoles. There was a statistically significant reduction for peak and average Medial Gastrocnemius muscle activity and peak Tibialis Anterior with insole trials While there was a reduction in average Tibialis Anterior muscle activity, the level was not statistically significant. Reducing lower leg muscle activity would have an accumulating beneficial effect for employees throughout daily, weekly, and annual work tasks. The greatest potential may be realized for those engaged in the utilities, transportation, hospitality, and service sectors of the economy.

Keywords: Gastrocnemius Muscle; Tibialis Anterior; Pain; Stiffness; Functional Limitation; Blood Vessels; Nerve Tissues

\section{Introduction}

Employees who work in static standing positions or associated work postures often complain of foot, knee, hip, and low back pain [1]. The reported pain responses can be a concern as employees' age and/or workload demands increase [2]. Static postures often generate isometric muscle contractions that can cause a reduction in returning blood flow affecting surrounding tissues, and can result in swelling in the lower extremities. Sanders [3] reported Kroemer \& Grandjean's research findings that reduced blood flow of the involved muscle tissues can decrease oxygen exchange and the removal of cellular by-products, leading to muscle fatigue and pain when the blood flow is constricted in proportion to the exertion and duration 


\section{Ergonomics International Journal}

of forces.

It is widely understood that organizations would be operationally and fiscally unable to put floor mats throughout a facility. One cogent strategic alternative to floor mats would be to establish a polyurethane insole program to address discomfort levels of employees associated with static standing or walking on concrete surfaces. Issued insoles offer employees the benefit of cushioning wherever they walk or stand in the facility, since the insole is in their respective footwear. Fang, et al. [4] found that an appropriately designed shoe insole can reduce pain, stiffness, and functional limitation.

Barnes and Smith [5] found that insoles provide both an increased area of support for the foot while reducing impact loads transmitted through the foot and leg upon heel strike as the foot contacts ground reaction forces. Polyurethane insoles have been shown to have beneficial influences on balance reactions and postural adjustments in addition to less muscle activity [6]. Sanders [3] indicated that a reduction in postural muscle contraction forces during static standing could lead to a reduction of compression forces on blood vessels and nerve tissues. A reduction in compressive isometric muscle contraction forces can lead to improved circulation, enhanced removal of metabolic waste materials, beneficial influence on fatigue, and reduced postural discomfort over prolonged time periods.

\section{Methods}

The study at Hasbro Games objectively investigated peak and average EMG lower leg muscle activity of 43 manufacturing employees whose primary tasks involved walking, static, and dynamic standing postures ranging between 60 to 90 percent of their workday. The study cohort was comprised of 27 males and females ranging in age from 37 to 66 years old. All participants signed an informed consent form and understood the process for EMG data collection. The insoles used for the study were the same company-issued polyurethane insoles available in whole sizes. The insole did have a unique feature of partially molding to the employee's specific foot pressure profile.

The purpose of the study was to measure lower extremity muscle activity during walking, static, and dynamic standing using electromyography (EMG) of the right Tibialis Anterior and Medial Gastrocnemius muscles. A BIOPAC System MP36 Four Channel Data Acquisition device was used to monitor and record the subject's right Tibialis Anterior and Medial Gastrocnemius muscle. Peak and average EMG activity was compared to the participant's own maximum voluntary contraction (MVC). After the participants read and signed the institutional review board's approved informed consent, the subjects' MVC was recorded for both muscle groups without wearing insoles. A standard instruction was given to stand as quickly as they could on the tips of their toes for 15 seconds to obtain the MVC for the Medial Gastrocnemius. The subjects were then instructed to change positions to maintain balance on only their heels for 15 seconds as hard as they could possibly endure, to capture the MVC for the Tibialis Anterior. Researchers entering the EMG data were blinded as to the participants' trial for wearing or not wearing insoles until both trials were complete.

The surface electrodes were applied as instructed by the BIOPAC System manufacturer with the skin being prepared with a rigorous application of water-soaked paper towels to the intended electrode placement sites for effective abrasion of the skin surface. The skin was appropriately dried and the manufacturer's electrodes applied with the ground located over the medial aspect of the tibia for both the Tibialis Anterior and Gastrocnemius muscle sites. The remaining electrodes were securely applied to the appropriate muscle locations, and the entire right calf electrodes were secured with an ACE wrap to maintain the electrode placement and reduce the potential for artifact during data collection.

Once the MVC requirements and secured electrode placements were satisfied, the participant was asked to maintain a period of relaxed static standing for 30 seconds before beginning a standardized task that assimilated the physical activities of the workplace on the factory concrete floor. Participants were chosen randomly during their regular shift and tested with a new pair of the standard insoles from the company's established insole program. The simulated work activity involved a performance of reaching and lifting a 6-ounce weight, then carrying the object 10 feet and placing the 6-ounce weight on a waist-high counter. The participant again reached for the object and kept repeating the process continuously for a period of one minute on the factory floor. The simulated work activity was repeated again with the same distances and weighted object for the same length of time returning to a static standing position for a set 30 -second period before simulating the same worker activities.

The settings for the EMG data acquisition and analysis device were pre-established in the input channel parameters. The digital filters were set with one high pass filter at $30.00 \mathrm{~Hz}$ and a $\mathrm{Q}$ setting of 0.70700 . The hardware was set at a gain of 2500 , offset at $0.0 \mathrm{mV}$, input coupling for alternating current with $1 \mathrm{kHz} \mathrm{LP}$, and the unit parameters were set for EMG 30-500 $\mathrm{Hz}$ for both channels involving the medial head of the Gastrocnemius and Tibialis Anterior muscles. EMG 


\section{Ergonomics International Journal}

signal data were collected in raw EMG form and integrated form with a rectified average of more than 50 samples per second. Data were analyzed using the BIOPAC Lab Pro 3.6.7 version software for data analysis from the above-described EMG signals for both muscles for all of the participants in the same manner.

\section{Results}

Analysis of the EMG results revealed a decrease in peak and mean EMG activity for both lower leg muscle groups with the use of insoles. While Tibialis Anterior
EMG muscle activities with insoles use demonstrated lower muscle activity, only the peak muscle activity demonstrated a statistically significant (0.05 level) reduction in EMG muscle activity. However, peak and mean Gastrocnemius EMG muscle activity were reduced to a statistically significant ( 0.05 level) for trials using insoles. Participants' subjective responses were surveyed revealing $98 \%$ had subjectively reported reduction in discomfort levels at the feet, lower legs, hips, and low back while wearing the insoles since the start of the Hasbro insole program. Table 1, Figures 1-4, $\mathrm{n}=43$.

\begin{tabular}{|c|c|c|c|c|c|}
\hline \multicolumn{6}{|c|}{ Paired Samples Test - Paired Differences } \\
\hline Insoles vs. No Insoles Trial & $\begin{array}{l}\text { Mean EMG } \\
\text { Difference }\end{array}$ & Std. Dev. & 95\% Confideı & Difference & $\mathbf{t}$ \\
\hline Peak EMG Tibialis Anterior & -5.9037 & 5.41 & -7.571 & -4.237 & $-7.147^{*}$ \\
\hline Mean EMG Tibialis Anterior & -1.8142 & 2.37 & -2.546 & -1.082 & -5.004 \\
\hline Peak EMG Medial Gastroc & -4.5326 & 5.73 & -6.299 & -2.766 & $-5.179 *$ \\
\hline Mean EMG Medial Gastroc & -3.2605 & 3.27 & -4.269 & -2.251 & $-6.521^{*}$ \\
\hline
\end{tabular}

Table 1: Results of 43 subjects with the Paired T test of the insoles trial compared to no insoles trial for both leg muscle groups; peak and mean EMG activity (*Significant at 0.05 level).

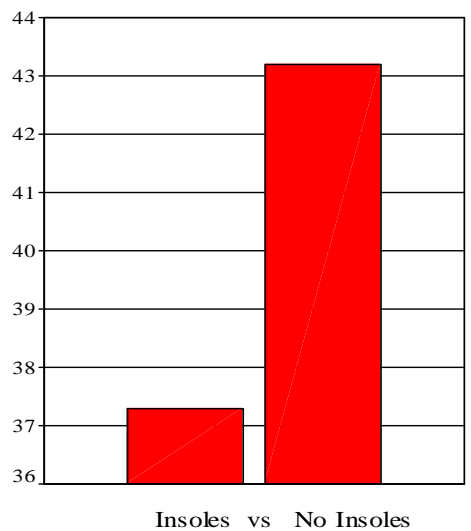

Figure 1: Comparison of Peak EMG Tibialis Anterior as a percent of MVC.

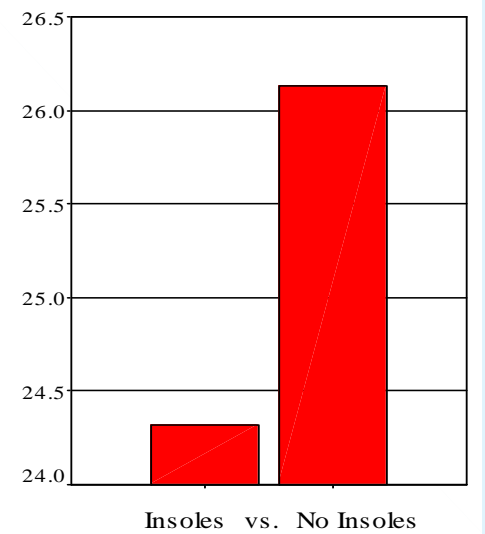

Figure 2: Comparison of Mean EMG Tibialis Anterior as a percent of MVC.

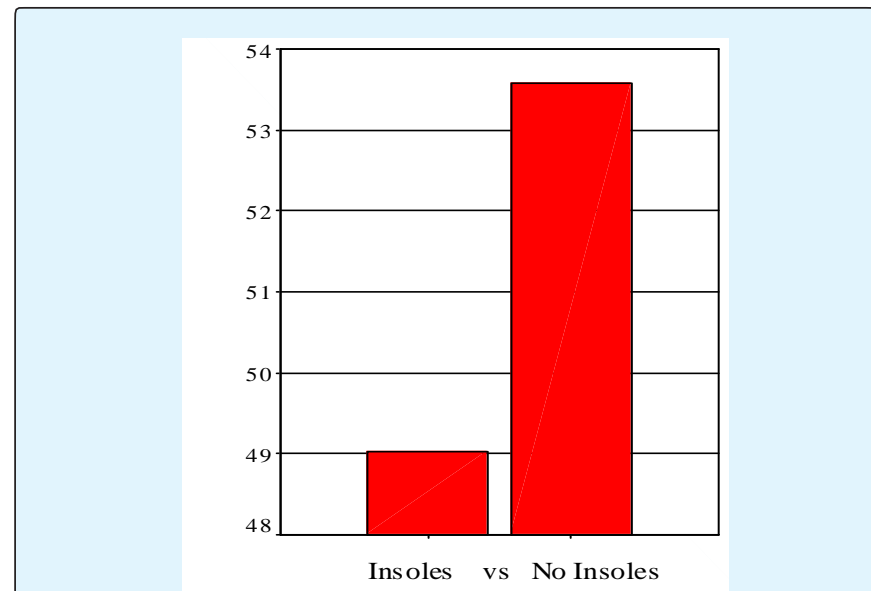

Figure 3: Comparison of Peak EMG Gastrocnemius as a percent of MVC.

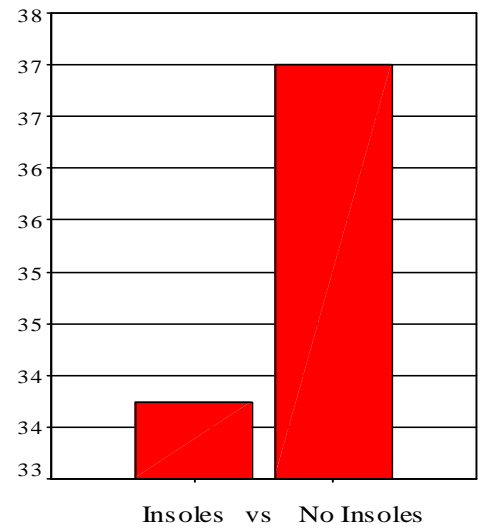

Figure 4: Comparison of Mean EMG Gastrocnemius as a percent of MVC. 


\section{Ergonomics International Journal}

\section{Discussion}

\section{Hypothesis Testing of the EMG Data}

The null hypothesis stated that there would be no difference in the means for either peak or average EMG activity for both the Tibialis Anterior (TA) and Medial Gastrocnemius (MG) muscles for those using insoles compared to those conditions without insoles. Based on the literature, an alternative hypothesis was proposed stating there would be less peak and mean EMG activity for both leg muscle groups for those wearing insoles. A paired one-tailed Student T test was used in comparing the means revealed that overall three out of the four conditions tested demonstrated a statistically significant difference in lowering leg muscle activity wearing insoles as opposed to those not wearing insoles (TA Peak $\mathrm{p}=4.48$, TA Mean $\mathrm{p}=5.26$, MG Peak $\mathrm{p}=2.97$, EMG MG Mean $\mathrm{p}=3.54$ ).

Fernberg [7] noted Ford's observation that the act of static standing alone is not the concern, but the small surface area of the foot is a concern relative to the pressure of body weight that is applied over a standard work shift. Workers will compensate for uncomfortable foot pressure by lifting up one leg on a higher surface or making momentary shifts in body weight from one side to the other. Meijsen and Knibbe [8] examined operating nurses standing for prolonged periods of time that resulted in additional positional compensation, swelling in the legs, and possible joint complications beyond muscle fatigue factors, noting that prolonged standing should be considered as an additional occupational risk factor influencing subjective complaints.

Fernberg [7] implied the effect of different floor surfaces that people work on will be magnified by the age and types of footwear worn. Prior studies on balance reactions, floor interfaces, and footwear choices have shown that excessive balance adjustments are observed with workers wearing older footwear, worn sneakers, and standing on soft rubber floor matting material [6]. In those studies, insoles shaped to accommodate the normal profile of their feet not only increased the foot surface contact, but also unexpectedly improved standing balance reactions, lowered maximal balance exertions, and statistically significant improvement in directional control. In a prior pilot study, those workers tested with work boots and contoured polyurethane insoles demonstrated the most efficient balance reaction profile in all age groups from 20 to 59 years old. Orlando and King [1] investigated the perceptions of standing discomfort that statistically described no "significant differences were found between flooring condition and subjective ratings of fatigue or discomfort in various body regions."

\section{Biomechanical Effects of Insole Design}

The design of insoles is important when considering most over-the-counter insoles are intended to add cushioning material without attempting to account for the foot's surface contours to maximize the potential biomechanical benefits. The more expensive over-thecounter polyurethane insoles will usually incorporate some type of heel cup and quasi-arch cushion design. However, due to the limited retail space available, each packaged insole will typically fit three to four shoe sizes with lines showing the consumer where to cut the excess insole material. However, trimming an insole to a smaller size causes the proportions of their respective heel cup and the location of the arch to become distorted against the foot's surface. The resulting location and size of these two components become displaced more forward, or the heel cup too big, when the consumer trims the insole by three to four sizes. For example, a worker who follows the recommended trimming [size 8-12] to a size 8 foot, will now experience a much larger heel cup area and arch cushion much more forward under the foot's surface. The larger size heel cup and location of the arch material for a size 12 is now disproportionate for someone with a smaller size.

The insoles worn in the study were from Hasbro's insole program supplied by Mega Comfort who provided whole sizes and no trimming needed for any of the insoles. As a result, the appropriate dimensions for heel cups and placement of arch cushioning were preserved and size specific with the requisite amount of polyurethane material based on the actual foot size, not on the available retail shelving space. The benefit of size specific insoles in suitable work footwear maintains subtalar joint orientation during static and dynamic standing positions. The ankle position with polyurethane cushioning permits necessary adjustments and cushioning influences that extended to the entire kinetic chain of the legs to the low back area based on early subjective studies [1]. The subjective responses of reduced ankle, knee, hip, and back discomfort in these earlier studies confirmed comparable outcomes of less discomfort reported in the low back area with a reduction in internal rotation of the tibia and femur, less anterior tilting of the pelvis, and a limited bias towards trunk flexion $[9,10]$.

\section{Polyurethane Insole Impact during the Gait Cycle}

The gait cycle consists of the stance phase and the swing phase shifting points of pressure against the foot surface [5]. The stance phase is comprised of three elements referred to as heel strike, single limb support, 


\section{Ergonomics International Journal}

and push off. During the stance phase, body weight is transferred from the heel to the forefoot as the foot transitions from pronation to supination [11]. During heel strike, Dixon [12] suggested the function of properly designed insoles could enhance the cushioning and limit subtalar eversion, thereby reducing the peak loading response by the Tibialis Anterior. Otherwise, excessive and repeated dynamic loading upon heel strike could create the likelihood of overuse injuries [12]. Previous studies have shown that the inversion/eversion movement of the foot is transferred through ankle joint into external/internal rotation of the tibia [13]. Therefore, weight bearing internal/external rotation of the tibia relative to femur is a primary kinematic variable affecting postural biased positions leading to potential injuries to the foot, knee, hip, and low back areas.

During the single limb support and the contralateral swing limb advancement component, the weightbearing foot rolls into a pronated position with both the forefoot and rear foot in contact with the ground as knee flexion gives way to hip and knee extension [5]. According to Cheung and $\mathrm{Ng}$ [11], the foot inverter muscles are the major rear-foot stabilizing muscles; if they become less efficient in controlling foot pronation, this will lead to higher plantar forces on the medial structures of the foot. Higher plantar force conditions can be related to overuse and fatigue of these stabilizing muscles. When the inverter muscles become fatigued, they become less efficient and the muscle tuning system becomes less responsive to external forces altering the movement patterns during standing balance and weight shifting [14].

The beneficial use of modified polyurethane insoles has been observed in the gait cycle and for the treatment of medial compartment knee osteoarthritis [4]. The insoles utilized in this study were designed with more material placed at the arch and surrounding the heel area with a top layer that would compress further improving the insole's conforming response to the foot profile. This was similar to another insole design that also provided added perceived comfort to workers [15]. The added cushioning around the heel cup area could influence calcaneal motion upon heel strike, thereby mitigating the opposing ground reaction force with less peak eccentric muscle activity from the Tibialis Anterior. The EMG study's data evidenced the statistically significant reduction of peak EMG recordings while wearing the insoles compared to no insoles. Overall, the reduction in muscle activity decreases the risk associated with standing fatigue thereby creating a biomechanically efficient advantage to the employee with the established company's insole program.

\section{Proprioceptive and Sensory Feedback of Properly Designed Insoles}

Previous researchers have agreed that the surface upon which an employee stands is a major contributing factor in the reported fatigue and their perception of discomfort related to prolonged standing $[1,8,16,17]$. Even in situations where excess ground reaction forces can be experienced, Wiggermann and Keyserling [18] reported shock-absorbing insoles can decrease selfreported pain among young soccer players training on artificial turf. Siwawut A [19] concluded that healthcare workers reported improvements after using commercially available insoles, which included muscle soreness, fatigue, foot pain, and general foot health.

In comparison, floor mats are commonly used to reportedly reduce fatigue; but their location under the footwear's surface and not directly under the person's foot surface places the floor mat at a considerable disadvantage for sensory and proprioceptive feedback necessary for efficient muscle and balance adjustments [6]. Nurse, et al. [20] stated, "Sensory feedback from the feet may affect specific motor unit pools during different activities. Changing the texture, without changing the geometry, of the shoe insert can alter muscle activity during walking." Additional researchers have described the same importance for the proprioceptive feedback of the feet to adjust for balance and posture. Pope, Goh, and Magnusson [21] stated, "In recent years, more attention has been given to the neural feedback system. Proprioception is an important factor in stabilization of the joints and throughout the spine. It is probable that proprioception deficits are associated with low back pain and low back injuries."

Polyurethane material for insoles is well adopted for protecting the body from potentially harmful and repeated impact by dampening the accumulative effect of ground reaction impact forces. The body will need to respond by more or less muscle control in constantly attenuating the opposing ground forces against the heels [5]. Hansen, Winkel, and Jorgensen [22] conducted an EMG study that determined a cushioned shoe provided a greater reduction in heel impact during static and dynamic positions with a corresponding reduction in postural muscle fatigue.

\section{Conclusion}

Prior to this study, little was known as to the physical effect shifting from floor mats to insoles. Most decisions were predicated more on subjective response of the employees. However, this study does provide objective physiological data supporting the efforts of a 


\section{Ergonomics International Journal}

polyurethane insole program throughout the facility. The results indicated statistically significant reductions in lower leg muscle activity at the workplace with insoles. Reductions of leg muscle activity understandably would have an accumulating beneficial impact on those in the workplace on a daily, weekly, and certainly, an annual basis. Insole programs offer a coherent, cost effective intervention not only for workplace static and dynamic standing, but also for those expected to walk noticeable distances during their workday.

The study also supports and encourages the continuation of Hasbro Game's insole program by demonstrating a reduction in lower leg muscle activity aligning with the subjective reduction in discomfort. The scope of this study did not investigate the potential results from other insole materials or various floor mats, since Hasbro Games was not interested in any comparisons for this particular effort. However, based on the insoles chosen by management, the objective EMG evidence supports the achievement of the insole programs intended purpose, which was to offer a lowcost alternative to the many floor mats while providing adequate comfort to their employees. With the demonstrated benefit of using appropriately designed insoles for standing and walking in the manufacturing environment, an insole program could easily be considered for those performing tasks in the utilities, transportation, healthcare, hospitality, and service sectors of the economy.

\section{Appreciation}

The researchers would like to thank Jack Popp, head of safety at Hasbro Games for his administrative support; Rosa DeRamos PhD, for her statistical assistance; the graduate physical therapy students at American International College; and Dr. Kevan Orvitz of MEGAComfort ${ }^{\circledR}$ for his support of additional insoles for the Hasbro Insole Program.

\section{References}

1. Orlando AR, King PM (2004) Relationship of demographic variables on perception of fatigue and discomfort following prolonged standing under various flooring conditions. Journal of Occupational Rehabilitation 14(1): 63-76.

2. Tanaka T, Takeda H, Izumi T, Ino S, Ifukube T (1999) Effects on the location of the centre of gravity and the foot pressure contribution to standing balance associated with aging. Ergonomics 42(7): 997-1010.

3. Sanders MJ (2004) Ergonomics and the management of musculoskeletal disorders. $2^{\text {nd }}$
(Edn.), Elsevier Health Sciences, ButterworthHeinemann.

4. Fang MA, Taylor CE, Nouvong A, Masih S, Kao KC, et al. (2006) Effects of footwear on medial compartment knee osteoarthritis. Journal of Rehabilitation Research \& Development 43(4): 427434.

5. Barnes RA, Smith PD (1994) The role of footwear in minimizing lower limb injury. Journal of Sports Sciences 12(4): 341-353.

6. Carley P, Lachowski S, Mullin E (2017) Floor mats and insoles: Workplace considerations for safe dynamic standing. Journal of Bones and Muscle Study 1: 1-8.

7. Fernberg PM (1999) Don't stand for fatigue. Occupational Hazards, 77.

8. Meijsen P, Knibbe HJ (2007) Prolonged standing in the or: A dutch research study. Aorn J 86(3): 399414.

9. Oatis C (2002) Kinesiology: The mechanics and pathomechanics of human movement. $1^{\text {st }}$ (Edn.), MD: Lippincott Williams \& Wilkins, Baltimore.

10. Razeghi M, Batt ME (2000) Biomechanical analysis of the effect of orthotic shoe inserts: A review of the literature. Sports Medicine 29(6): 425-438.

11. Cheung RT, Ng GY (2008) Influence of different footwear on force of landing during running. Physical Therapy 88(5): 620-628.

12. Dixon S (2007) Influence of a commercially available orthotic device on rearfoot eversion and vertical ground reaction force when running in military footwear. Military Medicine 172(4): 446450 .

13. Nigg BM, Khan A, Fisher V, Stefanyshyn D (1998) Effect of shoe insert construction on foot and leg movement. Medicine \& Science in Sports \& Exercise 30(4): 550-555.

14. Nigg BM (2001) The role of impact forces and foot pronation: A new paradigm. Clinical Journal of Sports Medicine 11(1): 2-9.

15. Carley P (1999) Healing the pain: Low-cost, size specific polyurethane shoe inserts help in managing perceived back and knee pain. Occupational Health and Safety 8(68): 92-94.

16. Madeleine $P$, Hoej BP, Fernández-de-Las-Peñas $C$ Rathleff MS, Kaalund S (2014) Pressure Pain 


\section{Ergonomics International Journal}

Sensitivity Changes After Use of Shock-Absorbing Insoles Among Young Soccer Players Training on Artificial Turf: A Randomized Controlled Trial. Journal of Orthopaedic \& Sports Physical Therapy 44(8): 587-594.

17. Hou JY, Shiao JS (2006) Risk factors for musculoskeletal discomfort in nurses. Journal of Nursing Research 14(3): 228-236.

18. Wiggermann N, Keyserling WM (2013) Effects of Anti-Fatigue Mats on Perceived Discomfort and Weight-Shifting During Prolonged Standing. Human Factors: The Journal of the Human Factors and Ergonomics Society 55(4): 764-765.

19. Siwawut A (2015) Formulated composite insoles reduced foot pain and fatigue and increase working efficiency in healthcare workers: A pilot non- controlled experimental study. British Journal of Medicine and Medical Research 5(10): 1294-1300.

20. Nurse MA, Hulliger $M$, Wakeling JM, Nigg BM, Stefanyshyn DJ (2005) Changing the texture of footwear can alter gait patterns. Journal of Electromyography and Kinesiology 15(5): 496-506.

21. Pope MH, Goh KL, Magnusson ML (2002) Spine ergonomics. Annual Review of Biomedical Engineering 4: 49-68.

22. Hansen L, Winkel J, Jørgensen K (1998) Significance of mat and shoe softness during prolonged work in upright position: Based on measurements of low back muscle emg, foot volume changes, discomfort and ground force reactions. Applied Ergonomics 29(3): 217-224. 